\title{
Dome-shaped microresonators and the Born-Oppenheimer method
}

\author{
Jens U. Nöckel ${ }^{a}$ and David H. Foster ${ }^{b}$ \\ ${ }^{a}$ Oregon Center for Optics, 1274 University of Oregon, Eugene, OR 97403-1274, USA; \\ ${ }^{b}$ Deep Photonics Corp., 5121 SW Hout St., Corvallis, OR, 97333, USA
}

\begin{abstract}
Numerical studies of the vector electromagnetic fields in plano-concave microresonators have recently revealed that a photonic analogue of spin-orbit coupling can occur in paraxial geometries. Laguerre-Gauss modes with circular polarization are then no longer the correct eigenstates, even if the resonator is axially symmetric. A crucial role in this effect is played by the presence of a boundary (e.g., a Bragg mirror) whose reflectivity at nonnormal incidence is polarization-dependent. Aiming for an analytical treatment that can incorporate both form birefringence and non-paraxiality, we explore the Born-Oppenheimer method as an alternative to the paraxial approximation. The conditions for the validity of these two approaches are different, but in a regime where they overlap we show that all the major results of paraxial theory can also be derived from the Born-Oppenheimer method. We discuss how this new approach can incorporate the Bragg stack physics in a way that can overcome the limitations of paraxial theory.
\end{abstract}

Keywords: Dome microresonator, Born-Oppenheimer approximation, paraxial approximation, Gaussian beam, Bragg reflector, polarization

\section{INTRODUCTION}

For applications of microresonators in quantum optics, it is essential to know the the spatial and spectral properties with a high degree of accuracy. For this purpose, we have developed a set of numerical methods ${ }^{1}$ that are able to calculate the vectorial electromagnetic fields in dome-shaped cavities with mixed boundary conditions over a large range of cavity shapes and size scales. In the present report, we focus on a geometry which at first sight appears to be treatable completely within the framework of standard paraxial theory, ${ }^{2}$ and which therefore was expected to be useful mainly for the purposes of validating the numerics.

For a theoretical treatment of dome-shaped cavities, the standard paraxial approximation provides a natural starting point, not least because the free-space modes to which the resonator ultimately couples are often Gaussian beams. However, as has been reported elsewhere, ${ }^{3}$ the high finesse achievable in realistic microcavities makes it possible to observe non-paraxial mode splittings which in larger resonators are washed out by the modal linewidths. We have studied the spectral fine structure that arises at frequencies well below the transverse mode splittings induced by the Gouy phase. This splitting of degenerate manifolds labeled by the transverse mode order $N$ of Gaussian-beam theory is due to the non-conservation of orbital angular momentum, even when the cavity has exact axial symmetry.

This is an effect that does not occur if all boundaries of an axially symmetric cavity are treated as ideal metals. But orbital angular momentum ceases to be conserved as soon as one of the boundaries is replaced by a Bragg mirror. The reason is that reflectivities at a dielectric interface are polarization dependent, and circularly polarized light is reflected from such an interface with elliptic polarization except at precisely normal incidence. This form birefringence amounts to coupling of orthogonal circular polarization states and indirectly translates into coupling of orbital angular momenta. This is qualitatively understandable within the paraxial approximation provided one retains the polarization information. However, the paraxial approximation has

Further author information: (Send correspondence to J.U.N.)

J.U.N.: E-mail: noeckel@uoregon.edu, Telephone: 15413465210

D.H.F.: E-mail: davidhfoster@gmail.com 
(a)

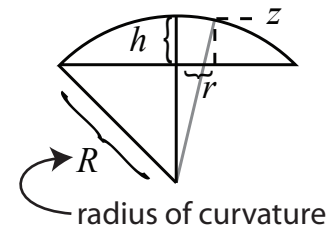

(b)

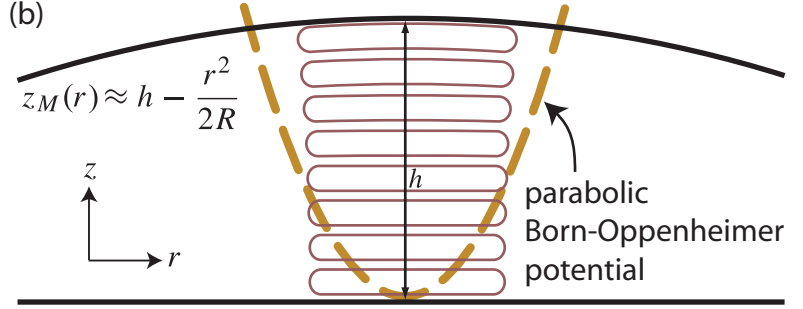

Figure 1. (a) Sketch of the cavity geometry. The vertical axis is $z$, and the radial distance from the $z$ axis is $r$. (b) Sketch of a paraxial mode; the Born-Oppenheimer potential $V_{B O}$ is repsonsible for the transverse confinement of the mode; it is created by the $r$-dependent cavity height, whose maximal value is $h$.

drawbacks for quantitive predictions, mainly because of the limited amount of detail it incorporates about varying dome curvature and angle-dependent reflectivity of the Bragg mirrors.

We have applied a different technique to the paraxial cavity problem, which is able to reproduce the results of paraxial Gaussian-beam theory when applied to constant-curvature mirrors with constant reflectivity, but can also applied when the latter assumptions are dropped. Our method is analogous to the Born-Oppenheimer approximation of molecular physics, ${ }^{4}$ and its most immediate benefit is to offer an alternative viewpoint of the Laguerre-Gauss beams and their relation to the two-dimensional harmonic oscillator, a topic of recent interest. ${ }^{5}$ In particular, our approach makes it straightforward to map mirrros with varying curvature onto anharmonic oscillators, and can even be applied to geometries that do not have an obvious beam axis (as found in axially symmetric cavities).

\section{ADIABATIC METHOD FOR THE HELMHOLTZ EQUATION}

As shown in Fig. 1, we consider a plano-convex resonator with axial symmetry around the $z$ axis. For definiteness, assume dimensions of $R=100 \mu \mathrm{m}$ and $h=10 \mu \mathrm{m}$. Although our numerical simulations take into account losses due to finite mirror transmittivity, we shall assume below that $|r|^{2}=1$, because the aim here is an approximate analytic treatment. For the same reason, we begin with the scalar Helmholtz equation and add polarization information below.

Because we think of $z$ as the beam axis, we write the wave equation as

$$
\begin{aligned}
\nabla^{2} \psi(\mathbf{x})+k^{2} \psi & =0 \\
\Rightarrow \frac{\partial^{2} \psi}{\partial z^{2}}+\nabla_{\perp}^{2} \psi(\mathbf{x})+k^{2} \psi & =0 .
\end{aligned}
$$

where $\nabla_{\perp}^{2}$ is the Laplacian in the $x y$ plane. We now solve this equation with the boundary conditions that the bottom mirror has a unimodular reflectivity $\exp (i \phi)$, and that the top mirror satisfies $\psi\left(x, y, z=z_{M}(x, y)\right)=0$. Here, $z_{M}(x, y)$ is the perpendicular cavity height as a function of the transverse coordinates $x$ and $y$.

The $z$ coordinate will assume a special role, but in contrast to the paraxial approximation, it will not enter the wave equation as a time-like variable. In particular, we will derive Gaussian-beam type modes without resorting to a parabolic differential equation. A sketch illustrating the type of cavity modes to be described is shown in Fig. 1 (b). In particular, there is a maximum cavity height $h$ which in a conventional cavity coincides with the location of the beam axis.

The central step is to assume with Born and Oppenheimer ${ }^{4}$ that the $x, y$ variation is of long wavelength, and use the envelope ansatz $\psi=\eta(z ; x, y) \chi(x, y)$ in the wave equation:

$$
\chi(x, y) \frac{\partial^{2} \eta(z ; r)}{\partial z^{2}}+\left(\frac{\partial^{2}}{\partial x^{2}}+\frac{\partial^{2}}{\partial y^{2}}\right) \eta(z ; x, y) \chi(x, y)+k^{2} \eta(z ; x, y) \chi(x, y)=0 .
$$


In the lowest (adiabatic) approximation, we neglect the dependence of $\eta$ on the parameters $x, y$. This allows us to separate off the equation for $\eta$ :

$$
\begin{aligned}
-\chi(x, y) \frac{\partial^{2} \eta(z ; x, y)}{\partial z^{2}} & =\eta(z ; x, y)\left(\frac{\partial^{2}}{\partial x^{2}}+\frac{\partial^{2}}{\partial y^{2}}\right) \chi(x, y)+\eta(z ; x, y) k^{2} \chi(x, y) \\
\Rightarrow-\frac{1}{\eta(z ; x, y)} \frac{\partial^{2} \eta(z ; x, y)}{\partial z^{2}} & \equiv k_{z}^{2}(x, y) \equiv \frac{1}{\chi(x, y)}\left(\frac{\partial^{2}}{\partial x^{2}}+\frac{\partial^{2}}{\partial y^{2}}\right) \chi(x, y)+k^{2}
\end{aligned}
$$

Here, $k_{z}$ has been introduced as the separation constant. Now we determine $k_{z}(x, y)$ by solving the first equation for a given $(x, y)$,

$$
\frac{\partial^{2} \eta(z ; x, y)}{\partial z^{2}}=-k_{z}^{2} \eta(z ; x, y)
$$

with the boundary conditions stated at the outset, i.e., $\eta\left(z_{M}(x, y), x, y\right)=0$. The solutions are of the form

$$
\eta(z ; x, y)=e^{-i k_{z} z}+e^{i \phi} e^{i k_{z} z}
$$

where $\phi$ and $k_{z}$ may depend on $x, y$ parametrically. For Dirichlet boundary conditions on the bottom mirror, we have $\phi=\pi=$ const. At a given $(x, y)$, the condition fixing $k_{z}$ is then

$$
0=\eta\left(z_{M} ; x, y\right)=e^{-i k_{z} z_{M}}+e^{i \phi} e^{i k_{z} z_{M}} \Rightarrow k_{z}=\frac{1}{z_{M}(x, y)}\left(\nu \pi+\frac{\pi}{2}-\frac{\phi}{2}\right)
$$

where $\nu$ is an integer. Returning to the $x, y$ equation, we get

$$
\begin{aligned}
\left(\frac{\partial^{2}}{\partial x^{2}}+\frac{\partial^{2}}{\partial y^{2}}+k^{2}\right) \chi(x, y) & =k_{z}^{2}(x, y) \chi(x, y) \\
& \approx \frac{1}{z_{M}^{2}(x, y)}\left(\nu \pi+\frac{\pi}{2}-\frac{\phi}{2}\right)^{2} \chi(x, y)
\end{aligned}
$$

Now we sort the terms into constant and $x, y$-dependent ones to get a form similar to the two-dimensional Schrödinger equation, with the units chose such that $\hbar^{2} \equiv 2$ and mass $M=1$ :

$$
\begin{aligned}
& -\left(\frac{\partial^{2}}{\partial x^{2}}+\frac{\partial^{2}}{\partial y^{2}}+k^{2}\right) \chi(x, y)+\left(\frac{1}{z_{M}^{2}(x, y)}-\frac{1}{h^{2}}\right)\left(\nu \pi+\frac{\pi}{2}-\frac{\phi}{2}\right)^{2} \chi(x, y) \\
= & \left(k^{2}-\frac{1}{h^{2}}\left(\nu \pi+\frac{\pi}{2}-\frac{\phi}{2}\right)^{2}\right) \chi(x, y) \\
= & K^{2} \chi(x, y),
\end{aligned}
$$

where the last line defines $K$ as a new constant that will be determined by the eigenvalue problem for $\chi$. Here, we have added a term proportional to $1 / h^{2}$ on both sides, producing on the left-hand side the term

$$
V_{B O}(x, y) \equiv\left(\frac{1}{z_{M}^{2}(x, y)}-\frac{1}{h^{2}}\right)\left(\nu \pi+\frac{\pi}{2}-\frac{\phi}{2}\right)^{2},
$$

which we shall call the Born-Oppenheimer potential. In summary, we have the equation

$$
-\left(\frac{\partial^{2}}{\partial x^{2}}+\frac{\partial^{2}}{\partial y^{2}}+k^{2}\right) \chi(x, y)+V_{B O}(x, y) \chi(x, y)=K^{2} \chi(x, y)
$$

Note, however, that the separation constant $K$ as defined in Eq. (6) is a true constant only if the reflection phase $\phi$ is a constant as well. 


\section{2D HARMONIC OSCILLATOR PROBLEM}

To clarify the meaning of $V_{B O}$ in (7) and to make contact with known results, we consider here a specific example geometry in which the top mirror is a spherical shell, see Fig. 1 (a): The gray hypothenuse of length $R$ then satisfies the equation

$$
R^{2}=r^{2}+\left(R-h+z_{M}\right)^{2} \Rightarrow z_{M}(r)=h+\sqrt{R^{2}-r^{2}}-R .
$$

For small radial distances from the axis (where $z_{M}=h$ ), this can be expanded to yield

$$
\begin{aligned}
& z_{M}(r) \approx h+R\left(1-\frac{r^{2}}{2 R^{2}}\right)-R=h-\frac{r^{2}}{2 R} \\
& \frac{1}{z_{M}^{2}(r)} \approx \frac{1}{h^{2}}\left(1+\frac{r^{2}}{h R}\right)
\end{aligned}
$$

The approximations here are based on $r \ll R$. Inserting the last result into $V_{B O}(x, y)$, we get the particular form

$$
\begin{aligned}
V_{B O}(r) & =\frac{r^{2}}{h^{3} R}\left(\nu \pi+\frac{\pi}{2}-\frac{\phi}{2}\right)^{2} \\
& \equiv \frac{1}{2} \Omega_{\nu}^{2} r^{2}
\end{aligned}
$$

which identifies $V_{B O}(r)$ as a harmonic-oscillator potential with minimum at $r=0$, and with a natural frequency

$$
\Omega_{\nu}=\sqrt{\frac{2}{h^{3} R}}\left(\nu \pi+\frac{\pi}{2}-\frac{\phi}{2}\right) .
$$

In other words, the frequency $\Omega_{\nu}$ characterizing the confining potential $V_{B O}(r)$ takes on discrete values labeled by the longitudinal quantum number $\nu$ of the mode, defined in Eq. (2). The paraxial Gaussian beams will arise as bound states of this potential $V_{B O}(r)$, by solving the transverse wave equation, Eq. (5):

$$
-\left(\frac{\partial^{2}}{\partial x^{2}}+\frac{\partial^{2}}{\partial y^{2}}+k^{2}\right) \chi(x, y)+\frac{1}{2} \Omega_{\nu}^{2} r^{2} \chi(x, y)=K^{2} \chi(x, y) .
$$

The harmonic-oscillator solutions in Cartesian coordinates are the Gauss-Hermite functions, appearing in the product form

$$
\chi_{\mu \mu^{\prime} ; \nu}(x, y)=\sqrt{\frac{2}{\pi w_{\nu}^{2} 2^{\mu+\mu^{\prime}} \mu ! \mu^{\prime} !}} H_{\mu}\left(\sqrt{2} \frac{x}{w_{\nu}}\right) e^{-x^{2} / w_{\nu}^{2}} H_{\mu^{\prime}}\left(\sqrt{2} \frac{y}{w_{\nu}}\right) e^{-y^{2} / w_{\nu}^{2}},
$$

where the harmonic-oscillator quantum numbers labeling the Hermite polynomials $H_{\mu}$ and $H_{\mu^{\prime}}$ are $\mu, \mu^{\prime}=0,1, \ldots$ The length $w_{\nu}$ appearing in this equation is related to our fictitious oscillator "frequency" $\Omega_{\nu}$ of Eq. (12) by

$$
\begin{aligned}
w_{\nu} & =\frac{2^{3 / 4}}{\sqrt{\Omega_{\nu}}} \\
& =\sqrt{2} \sqrt{\frac{\sqrt{h^{3} R}}{\nu \pi+\frac{\pi}{2}-\frac{\phi}{2}}}
\end{aligned}
$$

This is just the mode waist radius at the focus, as can be seen by noting that $w_{\nu}$ measures the exponential decay length of the harmonic-oscillator solutions (14).

The same result for the waist radius is obtained from paraxial theory in the limit $h \ll R$, starting from the textbook expression for the mode-waist radius

$$
w_{\text {paraxial }}=\sqrt{2}\left(\frac{h(R-h)}{k^{2}}\right)^{1 / 4} .
$$


Putting Eq. (14) back together with the $z$-dependence of the field as contained in $\eta(z ; x, y)$ from Eq. (1), we arrive at the standard Hermite-Gaussian modes,

$$
\mathrm{HG}_{\mu, \mu^{\prime} ; \nu}(x, y, z)=\chi_{\mu \mu^{\prime}}(x, y)\left(e^{-i k_{z}(r) z}+e^{i \phi} e^{i k_{z}(r) z}\right)
$$

This includes the downward- and a upward-propagating waves making up the mode. The correspondence between this result and the textbook form is established under the approximation $h \ll R$, and using the explicit form of $k_{z}(r)$ as defined in (2) with $z_{M}(r)$ given by $(9)$ :

$$
\begin{aligned}
k_{z}(r) & \approx \frac{1}{h}\left(1+\frac{r^{2}}{2 h R}\right)\left(\nu \pi+\frac{\pi}{2}-\frac{\phi}{2}\right) \\
& =\sqrt{\frac{h R}{2}} \Omega_{\nu}\left(1+\frac{r^{2}}{2 h R}\right)
\end{aligned}
$$

The $r$ dependence of $k_{z}$ due to the Born-Oppenheimer approximation now shows up as the wavefront curvature in Eq. (16).

The spectrum of $K$ in Eq. (13) is derived by simply transscribing the theory of the quantum mechanical 2D harmonic oscillator. We get

$$
\begin{aligned}
K_{\mu, \mu^{\prime} ; \nu}^{2} & =\sqrt{2} \Omega_{\nu}\left(\mu+\mu^{\prime}+1\right) \\
& =\frac{2}{\sqrt{h^{3} R}}\left(\nu \pi+\frac{\pi}{2}-\frac{\phi}{2}\right)\left(\mu+\mu^{\prime}+1\right) .
\end{aligned}
$$

From this we can obtain the transverse mode mode splitting by solving the definition of $K$ in Eq. (6) for the mode wave number $k$. We have the relation

$$
k_{\mu, \mu^{\prime} ; \nu}^{2}=K_{\mu, \mu^{\prime} ; \nu}^{2}+\frac{h R}{2} \Omega_{\nu}^{2}=K_{\mu, \mu^{\prime} ; \nu}^{2}+k_{z}^{2}(r=0) .
$$

The last equality arises from (18) with $r=0$. Taking the square root and assuming the transverse "energy" $K_{\mu, \mu^{\prime} ; \nu}^{2}$ to be much smaller than the longitudinal contribution $k_{z}^{2}(r=0)$, we get

$$
k_{\mu, \mu^{\prime} ; \nu} \approx \frac{1}{h}\left(\nu \pi+\frac{\pi}{2}-\frac{\phi}{2}\right)+\frac{2}{\sqrt{h R}}\left(\mu+\mu^{\prime}+1\right)=\sqrt{\frac{h R}{2}} \Omega_{\nu}+k_{G}\left(\mu+\mu^{\prime}+1\right)
$$

The last term is the Gouy phase contribution to the wave number,

$$
k_{G}=\frac{2}{\sqrt{h R}} .
$$

This result agrees with conventional resonator theory where the Gouy phase $\theta_{G}$ for a dome geometry is given by

$$
\cos \theta_{G}=1-\frac{2 h}{R}
$$

Since the radius of curvature $R$ is large, $\theta_{G}$ will be close to zero and we can use a small-argument expansion to get

$$
\theta_{G} \approx 2 \sqrt{\frac{h}{R}}
$$

This reduces to $k_{G}$ as defined above when divided by $h$ to turn the angle $\theta_{G}$ into a wavenumber.

A final property characterizing the modes is their "beam divergence", which in the case of a confined cavity really means the angular spectrum of their plane-wave content. Motivated by free-space optics, we first introduce the paraxiality parameter $\theta_{p}$. We define this to be the far-field divergence angle of the wave if continued to large 
$z$. The smaller the spreading angle, the more paraxial the beam is. For a Gaussian beam, this divergence angle is diffractively related to the beam waist, leading to

$$
\begin{aligned}
\theta_{p} & \equiv \arctan \frac{\lambda}{\pi w} \approx \frac{\lambda}{\pi w}=\frac{2}{k w}=\frac{\sqrt{\Omega_{\nu}}}{k} 2^{1 / 4} \\
& =\sqrt{2}\left(\frac{1}{h^{3} R}\right)^{1 / 4} \frac{1}{k} \sqrt{\nu \pi+\frac{\pi}{2}-\frac{\phi}{2}}
\end{aligned}
$$

Inserting the explicit expression (23) for the wavenumber of the quantized modes, one finds approximately

$$
\theta_{p} \approx \sqrt{2}\left(\frac{h}{R}\right)^{1 / 4} \frac{1}{\sqrt{\nu \pi+\frac{\pi}{2}-\frac{\phi}{2}}}
$$

if the transverse mode splitting proportional to $k_{G}$ is neglected in the denominator $(k)$. Thus, we can reduce the paraxiality parameter arbitrarily by either making $R$ large, $h$ small, or considering modes with high longitudinal quantum number $\nu$.

Since the modal wave number in (20) depends only on the sum $\mu+\mu^{\prime}$ of the harmonic-oscillator quantum numbers in the $x$ and $y$ directions, one introduces the mode order

$$
N \equiv \mu+\mu^{\prime}
$$

and writes

$$
k_{\nu, N} \approx \frac{1}{h}\left(\nu \pi+\frac{\pi}{2}-\frac{\phi}{2}\right)+k_{G}(N+1) .
$$

The first term is the Fabry-Pérot quantization, and the second term breaks the degeneracy of the logitudinal modes with equal number of nodes, $\nu$. The resulting splittings are small if $k_{G}$ is small. However, the last term still retains true degeneracies of multiplicity

$$
D_{N}=N+1
$$

Note that all modes in the degenerate manifold belonging to a given $N$ have the same value of $\theta_{p}$; this is true even if we do not neglect the the transverse mode splitting in $k$ due to the Gouy phase.

The calculations in this section have shown that the Born-Oppenheimer method is an alternative to the paraxial approximation for calculating all the relevant properties of a Gaussian beam. The two approaches become identical in the limit $h \ll R$, but the Born-Oppenheimer approach can be applied to more general geometries.

A useful property of the Hermite-Gaussians is that in a harmonic approximation, they remain the correct scalar eigenfunctions if axial symmetry is broken: in that case, $V_{B O}=V_{B O}(x, y)$ has different curvatures in the $x$ and $y$ directions, and the lowest-order correction is simply to introduce two different natural frequencies $\Omega_{\nu}^{(x)}$ and $\Omega_{\nu}^{(y)}$ for the independent harmonic oscillators making up $\chi_{\mu \mu^{\prime} ; \nu}(x, y)$ in (14). The degeneracy (24) is therefore completely broken if the axial symmetry of the resonator is destroyed.

As is well-known, the degeneracy (24) affords the freedom to orthogonally transform the degenerate GaussHermite solutions of order $N$ into an equally degenerate set of Gauss-Laguerre functions, using formulas explicitly given in Ref. 6

$$
\Psi_{p, l ; \nu}(r, \vartheta) \equiv \sqrt{\frac{2 p !}{\pi w_{\nu}^{2}(p+|\ell|) !}} \exp \left(-\frac{r^{2}}{w_{\nu}^{2}}\right)\left(2 \frac{r^{2}}{w_{\nu}^{2}}\right)^{|\ell| / 2} \mathrm{~L}_{p}^{|\ell|}\left(2 \frac{r^{2}}{w_{\nu}^{2}}\right) e^{i \ell \vartheta}
$$

where $r$ is the cylindrical radius, $\vartheta$ the azimuthal angle and $\mathrm{L}_{p}^{|\ell|}(u)$ is the associated Laguerre polynomial. Here, $\ell$ is the orbital angular momentum and $p$ the number of radial nodes in the transverse wave field. One finds that the mode order is related to these new quantum numbers by

$$
N=2 p+|\ell| .
$$




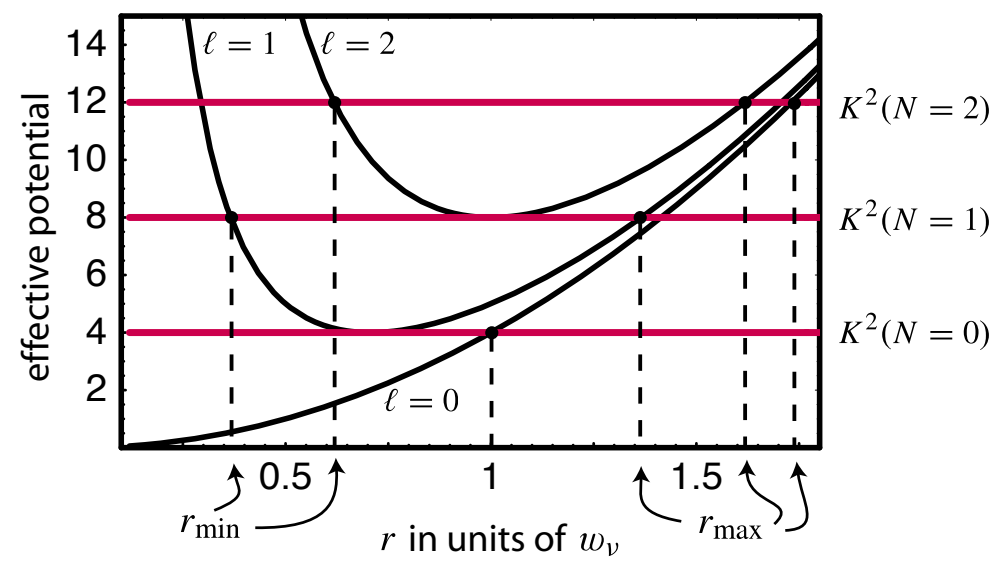

Figure 2. Effective potential for the Laguerre-Gauss modes, illustrating classical turning points and the role of $K^{2}$ as effective energy of the transverse oscillation. Shown as horizontal lines are three values of $K^{2}$ for mode orders $N=0,1,2$. The three curves labeled by orbital angular momentum $\ell=0,1,2$ represent the effective radial potential $\ell^{2} / r^{2}+\Omega^{2} r^{2} / 2$ of the oscillator. They intersect the lines $K^{2}=$ const at inner and outer turning points $r_{\min }, r_{\max }$ of the Laguerre-Gauss modes with a given $N, \ell$; the intersections corresponding to allowed modes are marked by dots. Dashed vertical lines indicate turning points. The points of tangency between the minima of the effective potential and the $K^{2}=$ const lines belong to $\ell=N+1$ which falls outside the range $\ell=-N, \ldots N$ of allowed LG modes.

The allowed angular momenta are $\ell=-N,-N+2, \ldots N-2, N$, with the highest value $|\ell|=N$ occuring when $p=0$. The fact that $\ell$ varies in steps of $\Delta \ell=2$ guarantees that the degeneracy of the Laguerre-Gaussians is again given by Eq. (24), as it must. In the same way that the Cartesian solutions lead to the definition (16), we obtain the Born-Oppenheimer version of the Laguerre-Gauss modes for our dome cavity as

$$
\operatorname{LG}_{\ell ; \nu}^{p}(r, \vartheta, z)=\Psi_{p, l ; \nu}(r, \vartheta)\left(e^{-i k_{z}(r) z}+e^{i \phi} e^{i k_{z}(r) z}\right)
$$

The radial part of the two-dimensional harmonic oscillator wave function $\Psi$ satisfies the equation

$$
-\left(\frac{\partial^{2}}{\partial r^{2}}+\frac{1}{r} \frac{\partial}{\partial r}\right) \Psi+\left(\frac{\ell^{2}}{r^{2}}+\left(\nu \pi+\frac{\pi}{2}-\frac{\phi}{2}\right)^{2} \frac{r^{2}}{h^{3} R}\right) \Psi=K^{2} \Psi,
$$

where $K_{N ; \nu}^{2}$ is the same as in (13). The first term is the kinetic energy and the second term the effective radial potential, which differs from $V_{B O}$ by the characteristic centrifugal potential $\ell^{2} / r^{2}$. This effective potential is shown Fig. 2; it depends both on $\nu$ and on $\ell$, but not on $N$.

Looking back at the Born-Oppenheimer derivation of the Gaussian profile for the scalar cavity fields, we made additional approximations beyond the Born-Oppenheimer condition in order to arrive at the correspondence between the transverse wave equation and the two-dimensional harmonic oscillator. The goal was to retain only quadratic $r$ dependencies in the wave equation. This step, which is how we made contact with the paraxial approximation, also has the immediate consequence of making the wave equation separable in Cartesian coordinates along the principal axes of the potential $V_{B O}(x, y)$, even if the mirror curvatures are not axially symmetric.

However, two effects could force us to abandon the harmonic approximation: (a) sufficiently curved mirrors can cause a breakdown of the quadratic approximation in Eq. (10); (b) the reflection phase $\phi$ of the planar bottom mirror may not be a constant and instead depend strongly on $r$. Case (b) can occur when the planar mirror is a Bragg stack and the cavity modes are not fully paraxial. As will be argued in the next section, this can be described within the Born-Oppenheimer approximation, by letting $\phi=\phi(r)$ in Eq. (5); the resulting problem may still be harmonic if $\phi(r)$ is at most quadratic in $r$, but a general reflectivity profile will not terminate at order $r^{2}$. 
If we thus keep terms of higher order in $r^{2} / h R$ in the potential $V_{B O}$ of Eq. (11) or in the phase $\phi$, we will introduce cross terms that break separability of Eq. (8) in Cartesian coordinates; this arises even in a simple potential term like $r^{4}$ which still has axial symmetry. Consequently, it is problematic to consider HermiteGauss beams and Laguerre-Gauss beams as exchangeable candidates for the description of modes in an axially symmetric cavity: As soon as we are concerned with anharmonicities, whether in axially symmetric cavities or not, the $\mathrm{HG}_{\mu, \mu^{\prime} ; \nu}$ modes of Eq. (16) are no longer solutions of the resonator problem. On the other hand, the Laguerre-Gaussians are closer to the true solution in an anharmonic cavity geometry as long as axial symmetry remains. The main reason is that the LG beams are symmetrized according to the irreducible representations of the two-dimensional rotation group $\mathrm{SO}(2)$, which is the symmetry group that leaves the resonator geometry invariant. Anharmonicities which preserve axial symmetry can be incorporated into our Born-Oppenheimer potential picture without difficulty: in Fig. 2, the curves representing the effective radial potential will change shape, but the physical picture of a one-dimensional well with discrete bound states remains unchanged.

\section{RELATION TO BESSEL-WAVE EXPANSION}

From a group-theoretical point of view, the degeneracy in Eq. (24) is "accidental", since all its members are labeled by different values of the conserved azimuthal angular momentum $\ell$; this is a well-known feature of the two-dimensional harmonic oscillator, which possess a hidden $\mathrm{SU}(2)$ symmetry ${ }^{4}$ where $N / 2$ plays the role of a fictitious total total angular momentum. This "hidden" symmetry is not even broken when we allow the reflection phase $\phi$ in Eq. (1) to depart from constancy, as long as $\phi(r)=\phi_{0}+\alpha r^{2}$. However, if we take this idea seriously, it must be kept in mind that the reflectivity of a realistic mirror is polarization-dependent. This dependence vanishes at normal incidence, but may become significant at nonzero angles of incidence. We first discuss how the scalar considerations outlined above can be combined to describe the degeneracy breaking of the vectorial modes in terms of two possible orthogonal polarizations. Then we address the question of how a dependence of reflection phase $\phi$ on incident angle translates into a dependence $\phi(r)$ of reflection phase on position in the Born-Oppenheimer equation.

Scalar fields are often a good approximation to the vector modes of a paraxial resonator, especially when the geometric length scales are much larger than the wavelength. In an axially symmetric cavity, the scalar wave equation emerges as soon as one makes the assumption that all cavity modes can be written as products of constant circular polarization vectors and scalar orbital parts. Using the Jones vectors $\sigma^{ \pm}=(1, \pm i) / \sqrt{2}$ to denote right- and left-circular polarizations, respectively, one would then obtain Eq. (26) for the orbital wave functions, and consequently expect a degeneracy given by (24), multiplied by two to account for the two orthogonal polarization states $\sigma^{ \pm}$. The vector Laguerre-Gauss beams are simply of the form $\mathrm{LG}_{\ell}^{p} \sigma^{ \pm}$.

However, these simplifying assumptions can break down even in a paraxial geometry, when the boundary conditions lead to coupling between different polarization states. As was noted in Ref. 3 this requires only the presence of a dielectric Bragg mirror, and a sufficiently high finesse so as to resolve the resulting lifting of the degeneracy (24). It is a rigorous result that the vectorial modes of a dome cavity bounded by a dielectric Bragg stack do not in general have a well-defined orbital angular momentum $\ell$, even when the resonator geometry is axially symmetric. Instead, only the total angular momentum $m$ is a conserved quantity; it differs from $\ell$ by the photon spin $s= \pm 1$ characterizing the circular polarization state of a vector Laguerre-Gauss beam.

If two members of the original degenerate manifold (24) labeled by $N$ have orbital angular momenta $\ell_{-}=m-1$ and $\ell_{+}=m+1$, their radial node numbers are $p_{-}=\left(N-l_{-}\right) / 2$ and $p_{+}=\left(N-l_{+}\right) / 2$. Since $\ell$ ranges over the set $\ell \in\{-N,-N+2, \ldots, N-2, N\}$, one has $m \in\{-N-1,-N+1, \ldots, N-1, N+1\}$. The true cavity modes are no longer degenerate if they have different $|m|$. Modes with the same $|m|$ but differing only in the sign of $m$ transform into each other under reflection and hence remain degenerate. See section 5 of Ref. 1 for a detailed

discussion; the analysis shows that cavity modes can typically be written as pairwise superpositions of $\mathrm{LG}_{\ell_{ \pm}}^{p_{ \pm}} \sigma^{\mp}$ waves with different orbital angular momenta, $\ell=\ell_{ \pm}$as defined above, and opposite circular polarization $\sigma^{\mp}$. If we denote the two possible orthogonal, non-degenerate superposition states as having (complex) electric field vectors $\mathbf{E}_{A}$ and $\mathbf{E}_{B}$, we can write their relation to the degenerate basis functions $\mathrm{LG}_{\ell_{ \pm}}^{p_{ \pm}} \sigma^{\mp}$ in matrix form as

$$
\left(\begin{array}{c}
\mathbf{E}_{A} \\
\mathbf{E}_{b}
\end{array}\right)=\left[\begin{array}{cc}
\cos \alpha & -\sin \alpha \\
\sin \alpha & \cos \alpha
\end{array}\right]\left(\begin{array}{c}
\mathrm{LG}_{\ell_{-}}^{p_{-}} \sigma^{+} \\
\mathrm{LG}_{\ell_{+}}^{p_{+}} \sigma^{-}
\end{array}\right)
$$




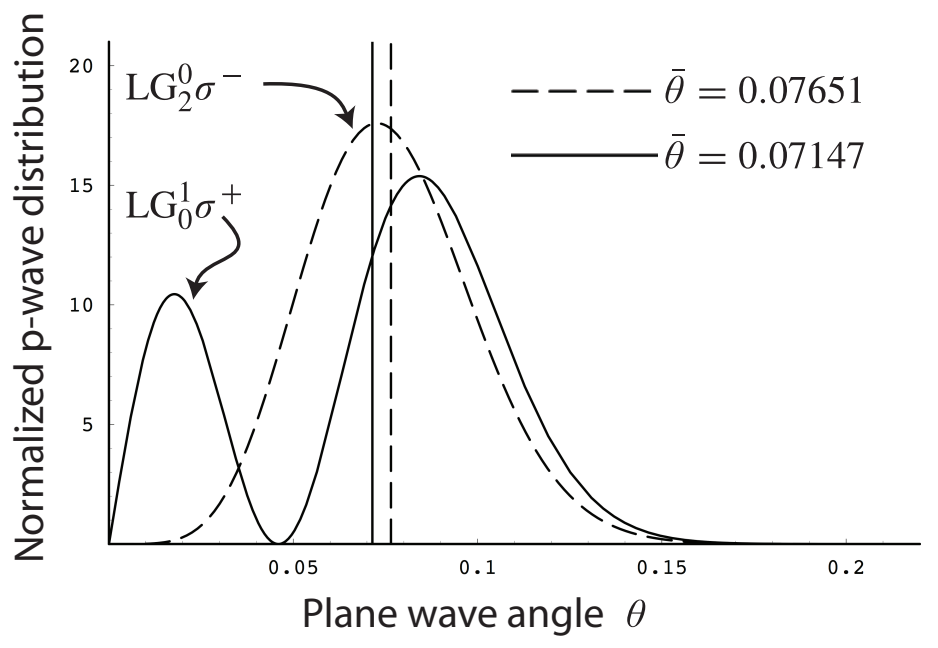

Figure 3. Angular spectrum $\sin \theta g(\theta)$ of two vector Laguerre-Gauss beams, $\mathrm{LG}_{2}^{0} \sigma^{-}$(dashed) and $\mathrm{LG}_{0}^{1} \sigma^{+}$(solid), obtained by applying a Bessel-wave decomposition to the free-space travelling-wave result of standard paraxial theory. Both beams are chosen to have paraxial spreading angle of $\theta_{p}=0.065$. Only the weight of the p-polarized Bessel wave component is plotted. Vertical lines indicate the average plane-wave angles $\bar{\theta}$ of each wave; note that the averages are unequal, and both are larger than the common $\theta_{p}$.

where $\alpha$ is a mixing angle. In Ref. 3, we studied in particular the case $N=2$ and $m=1$, where $\ell_{-}=0$ and $\ell_{+}=2$. Such a pairwise superposition of LG modes does not lead back to the Hermite-Gauss solutions, because that transformation typically requires all $N+1 \mathrm{LG}$ waves (not just two of them) to create a HG pattern. The polarization of the doublet modes is not strictly homogeneous, but numerically one finds that $\mathbf{E}$ is approximately linearly polarized, except at certain parameter combinations (varying cavity height $h$, dome radius $R$ or Bragg stack design) where the doublet splitting reaches a minimum. These are points of avoided crossings in the parameter space, and they are characterized by $\alpha=0$, leading to purely circular polarizations, $\mathbf{E}_{A, B}=\mathrm{LG}_{\ell_{ \pm}}^{p_{ \pm}} \sigma^{\mp}$.

The physical reason why this coupling between $\mathrm{LG}_{\ell_{+}}^{p_{ \pm}} \sigma^{\mp}$ in general leads to nonzero mixing angle $\alpha \neq 0$ is that even a paraxial beam has plane waves with non-normal angles of incidence in its angular spectrum. A Bragg stack in turn imparts a different reflection phase on plane waves with different angles of incidence. But the planewave content of the two $\mathrm{LG}_{\ell_{ \pm}}^{p_{ \pm}} \sigma^{\mp}$ components is different, owing to their different orbital angular momentum. Therefore, the $\mathrm{LG}_{\ell_{+}}^{p_{ \pm}} \sigma^{\mp}$ waves cannot be expected to remain degenerate. To illustrate this, Fig. 3 shows the angular spectrum of the vector Laguerre-Gauss beams with $N=2, m=1$, and $\ell_{-}=0, \ell_{+}=2$, relevant to Ref. 3.

To obtain a distribution $g(\theta)$ in terms of plane-wave angles $\theta$, we start with a plane wave $\mathbf{E}_{\theta}^{s / p}$ at incident angle $\theta$ and linear polarization state $s$ or $p$ with respect to the plane of incidence at the bottom Bragg mirror; from this wave, one obtains a Bessel beam $\mathrm{J}_{m, \theta}^{s / p}$ of angular momentum $m$ by symmetrization ${ }^{1}$; its electric field in the $x y$ plane is

$$
\mathrm{J}_{m, \theta}^{s / p}(r, \vartheta) \propto[J_{\ell_{-}}(k r \sin \theta) e^{i \ell_{-} \vartheta} \sigma^{+} \underbrace{ \pm}_{+ \text {for } s,- \text { for } p} J_{\ell_{+}}(k r \sin \theta) e^{i \ell_{+} \vartheta} \sigma^{-}] e^{i k r \cos \theta},
$$

where $\ell_{ \pm}$is defined as above, $J_{\ell}$ is a Bessel function, and $\vartheta$ denotes the azimuthal angle, as in Eq. (26). In contrast to the circularly polarized $\mathrm{LG}_{\ell_{ \pm}}^{p_{ \pm}} \sigma^{\mp}$ beams, the Bessel beam $\mathrm{J}_{m, \theta}^{s}$ acquires a well-defined phase shift $\phi=\phi_{s}(\theta)$ upon reflection at the Bragg mirror; similarly, a phase shift $\phi=\phi_{p}(\theta)$ applies to $\mathrm{J}_{m, \theta}^{p}$. Each LaguerreGauss beam can be written as a superposition of $\mathrm{J}_{m, \theta}^{s}$ and $\mathrm{J}_{m, \theta}^{p}$ with fixed $m$ but varying $\theta$, and the squared expansion coeffient of $\mathrm{J}_{m, \theta}^{p}$ is what we plot in Fig. 3 (weighted by a factor $\sin \theta$ to account for solid angle, so 
that $\int_{0}^{\pi} g(\theta) \sin \theta d \theta=1$ ). Because of the appearance of $\sigma^{+}$and $\sigma^{-}$in Eq. (28), a circularly polarized wave must contain both $\mathrm{J}_{m, \theta}^{p}$ and $\mathrm{J}_{m, \theta}^{s}$; in free space, their expansion coefficients must in fact be equal or opposite (disregarding normalization constants), causing only the scalar Bessel functions of one single angular momentum (either $\ell_{-}$or $\ell_{+}$) to appear. To understand the degeneracy lifting of the $\mathrm{LG}_{\ell_{ \pm}}^{p_{ \pm}} \sigma^{\mp}$, we therefore have to first address the simpler problem of decomposing the scalar functions $\mathrm{LG}_{\ell_{ \pm}}^{p_{ \pm}}$into scalar Bessel waves.

While Fig. 3 clearly shows the differing angular spectra of two originally degenerate modes, it also suggests that the average plane-wave angle $\bar{\theta}$ of the distribution does not capture much of its detail since there may be several maxima and minima. In order to understand the degree of Bragg-induced coupling between the two LG beams, we therefore should attempt to characterize the angular distributions $g(\theta)$ further. One important result is that $g(\theta)$ for a circularly polarized LG beam (as well as for a scalar LG beam) has the same functional form as the radial wave function $\left|\Psi_{p, l ; \nu}(r)\right|^{2}$ in (25), with the substitution $r=w_{\nu} \theta / \theta_{p}$ :

$$
g(\theta) \sin \theta \propto \sin \theta\left[\exp \left(-\frac{\theta^{2}}{\theta_{p}^{2}}\right)\left(2 \frac{\theta^{2}}{\theta_{p}^{2}}\right)^{|\ell| / 2} \mathrm{~L}_{p}^{|\ell|}\left(2 \frac{\theta^{2}}{\theta_{p}^{2}}\right)\right]^{2}
$$

This expression, derived in Ref. 7 within the paraxial approximation, is valid for small $\theta_{p}, \theta$.

We now want to investigate the duality $r \leftrightarrow \theta$ and $w_{\nu} \leftrightarrow \theta_{p}$ from the Born-Oppenheimer point of view, in order to address how the presence of the cavity affects the angular distribution $g(\theta)$. A starting point is provided by observing that Bessel waves are solutions to the Born-Oppenheimer wave equation, Eq. (8), in the limit of flat mirrors, $z_{M} \equiv h$, equivalent to $R \rightarrow \infty$ in Eq. (27). At finite mirror curvature, the Born-Oppenheimer approximation relies on the variation of $z_{M}(r)$ with $r$ being slow, ultimately to introduce a position-dependent wave number $k_{z}(r)$, cf. Eq. (1). Comparing the $z$-dependence of the traveling-wave components in Eq. (26) with Eq. (28), we make the local plane wave approximation

$$
\cos \theta(r)=\frac{k_{z}(r)}{k}
$$

This constitutes a local approximation because it interprets $\theta$ as the angle of the cone corresponding to the Bessel wave that we would obtain if the cavity had the same height $z_{M}(r)$ for all $r$.

For the wave number component $k_{z}(r)$, we use Eq. (18). Since most of the "energy" of the modes resides in the longitudinal motion, we can approximate

$$
\begin{aligned}
\theta(r) & =\arccos \frac{k_{z}(r)}{k} \approx \sqrt{2 \frac{k-k_{z}(r)}{k}} \\
& =\sqrt{\frac{2}{k}} \frac{1}{(h R)^{1 / 4}} \sqrt{2(N+1)-\left(\frac{r}{w_{\nu}}\right)^{2}} \\
& =\theta_{p} \sqrt{2(N+1)-\left(\frac{r}{w_{\nu}}\right)^{2}}
\end{aligned}
$$

Here, we have inserted the definition of the far-field spreading angle $\theta_{p}$ from Eq. (21). The Bessel-wave limit, $\theta \equiv$ const, is recovered from this when $w_{\nu} \rightarrow \infty$. Although this limit makes $\theta_{p}$ go to zero, we can get finite $\theta$ by simultaneously choosing $N$ large. The term containing $N$ in Eq. (31) originates from the transverse mode splitting $k_{G}(N+1)$ of Eq. (23), which depends on the Gouy phase $\theta_{G}$.

Equation (31) is the relation between $r$ and $\theta$ that we aimed for, motivated by Eq. (29). This expression can now be inserted into the Bragg reflectivity to express its phase as $\phi(r)=\phi(\theta(r))$. This is the crucial step allowing us to treat the Bragg stack in the Born-Oppenheimer approximation.

Clearly, this expression can be valid only for a limited range of $r$, because the square root becomes imaginary for large $r$. As illustrated in Fig. 2, there is in fact a well-defined interval of allowed $r$, delimited by the classical turning points in the effective radial potential. In particular, the angle $\theta$ is largest when $r$ takes on its smallest value, $r_{\min }$. This value depends on the angular momentum of the mode, as we expect from the well-known 
transverse donut shape of higher- $\ell$ Laguerre-Gauss beams where the axis $r=0$ is avoided. The calculation of $r_{\min }$ and $r_{\max }$ is straightforward; it amounts to reading off the intersections of the potential curves in Fig. 2 with the lines of $K^{2}=$ const, and inserting the results into Eq. (31). The inner and outer turning points of the LG modes with $\ell=0,1,2$ are illustrated by the vertical dashed lines in Fig. 2 . For $\ell=N=0$, we get $r_{\text {min }}=0$ and $r_{\max }=w_{\nu}$, the conventional mode waist radius.

However, whereas (29) suggests the simple proportionality $r=w_{\nu} \theta / \theta_{p}$, Eq. (31) predicts a monotonic decrease of $\theta$ with increasing $r$. This difference is physically significant: e.g., for $N=\ell=0$ we find that there is a finite angle of incidence $\theta=\sqrt{2} \theta_{p}$ at $r=0$. In the Born-Oppenheimer approach, the smallest angle of incidence with respect to the normal occurs at large radial distance from the axis. This is what one also expects from the ray picture. For the purposes of finding $\phi(r)$ as required for a Born-Oppenheimer treatment of the Bragg stack, we therefore have to use Eq. (31).

\section{CONCLUSION}

The adiabaticity assumption inherent in the Born-Oppenheimer approximation is suitable for a wide range of cavity geometries, including even VCSELs. We have explored this method with the motivation of addressing the optical analogue of spin-orbit coupling which we observed numerically in a paraxial dome resonator. ${ }^{3}$ This has led us to reconsider standard paraxial resonator optics from the Born-Oppenheimer point of view, providing alternative and efficient derivations of key results from Gaussian beam theory. The flexibility of our analytic method shows itself in particular in the possibility of including variable Bragg reflectivities. For this purpose, we derived a local relation for the Bessel-wave angle of incidence as a function of position on the Bragg mirror. Because we focused on the near-paraxial regime, we describe the breaking of transverse degeneracies due to spinorbit coupling in the basis of vector Laguerre Gauss modes, which in turn allowed us to make contact with the physics of the 2D harmonic oscillator. In the Born-Oppenheimer approach, further extentions of this description both to non-paraxial and to non-axially symmetric geometries can be implemented in an appealingly seamless way.

\section{ACKNOWLEDGMENTS}

This work was supported by NSF Grant ECE-02-39332.

\section{REFERENCES}

1. D. H. Foster and J. U. Nöckel, "Methods for 3-d vector microcavity problems involving a planar dielectric mirror," Opt. Commun. 234, pp. 351-383, 2004.

2. A. E. Siegman, Lasers, University Science Books, Sausalito, CA, 1986.

3. D. H. Foster and J. U. Nöckel, "Bragg-induced orbital angular-momentum mixing in paraxial high-finesse cavities," Opt. Lett. 29, pp. 2788-2790, 2004.

4. J. Elliot and P. Dawber, Symmetry in Physics, Vol. 2, Oxford University Press, New York, NY, 1990.

5. O. Steuernagel, "Equivalence between focused paraxial beams and the quantum harmonic oscillator," Am. J. Phys. 73, pp. 625-629, 2005.

6. S. Danakas and P.K.Aravind, "Analogies between two optical systems (photon beam splitters and laser beams) and two quantum systems (the two-dimensional harmonic oscillator and the two-dimensional hydrogen atom," Phys. Rev. A 45, pp. 1973-1977, 1992.

7. D. H. Foster, PhD thesis, University of Oregon, 2006. 